\title{
Counting visual and tactile events: The effect of attention on multisensory integration
}

\author{
Peter J. Werkhoven \\ Utrecht University, Utrecht, The Netherlands \\ JAN B. F. VAN ERP \\ TNO Human Factors, Soesterberg, The Netherlands \\ AND \\ ToM G. PhILIPPI \\ Utrecht University, Utrecht, The Netherlands
}

\begin{abstract}
Irrelevant events in one sensory modality can influence the number of events that are perceived in another modality. Previously, the underlying process of sensory integration was studied in conditions in which participants knew a priori which sensory modality was relevant and which was not. Consequently, (bottom-up) sensory interference and (top-down) selective attention were confounded. We disentangled these effects by measuring the influence of visual flashes on the number of tactile taps that were perceived, and vice versa, in two conditions. In the cue condition, participants were instructed on which modality to report before the bimodal stimulus was presented. In the no-cue condition, they were instructed after stimulus presentation. Participants reported the number of events that they perceived for bimodal combinations of one, two, or three flashes and one, two, or three taps. Our main findings were that (1) in no-cue conditions, the influence of vision on touch was stronger than was the influence of touch on vision; (2) in cue conditions, the integration effects were smaller than those in no-cue conditions; and (3) irrelevant taps were less easily ignored than were irrelevant flashes. This study disentangled previously confounded bottom-up and top-down effects: The bottom-up influence of vision on touch was stronger, but vision was also more easily suppressed by top-down selective attention. We have compared our results qualitatively and quantitatively with recently proposed sensory-integration models.
\end{abstract}

Various studies on human perception of incongruent sensory inputs have determined how one sensory channel is biased by task-irrelevant stimuli in another sensory channel. Shams, Kamitani, and Shimojo (2000, 2002) discovered the illusory flash effect, a "visual illusion that is induced by sound: When a single flash of light is accompanied by multiple auditory beeps, the single flash is perceived as multiple flashes." Andersen, Tiippana, and Sams (2004) extended this work by showing that the number of perceived flashes can be either increased (called fission) or decreased (called fusion) by presenting either a larger or a smaller number of irrelevant beeps in combination with the flashes. In addition to these audiovisual illusions, comparable effects have been reported for almost all other combinations of modalities (Bresciani et al., 2005; Courtney, Motes, \& Hubbard, 2007; Ernst, Bresciani, Drewing, \& Bülthoff, 2004; Hötting \& Röder, 2004; Violentyev, Shimojo, \& Shams, 2005).

Some studies have shown an asymmetry in bias effects. For example, Guest and Spence (2003) concluded that vision influences touch more than touch influences vision. This finding seems to support perceptual-integration models in which the more appropriate channel (i.e., most sensitive for the specific stimulus) dominates the less appropriate channel-in the most extreme form, the "winner-takes-all" model (see Welch \& Warren, 1980). Other studies (for a review, see Ernst \& Bülthoff, 2004) have found mutual influences that can best be explained by the assumption that sensory signals are integrated with weights that are inversely proportional to the relative signal-to-noise ratios (the relative reliabilities of the sensory channels). On the basis of this reliability assumption, various quantitative probabilistic "ideal observer" models have been developed to explicitly model multimodal perception. The maximum likelihood estimation (MLE) approach by Andersen, Tiippana, and Sams (2005) assumes that there is complete weighted integration of the sensory channels and, consequently, that the sum of the weights equals one. They found that early MLE (integration before stimulus categorization) explained the perceptual integration of rapid beeps and flashes better than did late MLE (integration after categorization).

Various sensory-integration studies (Bresciani, Dammeier, \& Ernst, 2006; Ernst \& Bülthoff, 2004; Körd-

P. J.Werkhoven, peter.werkhoven@tno.nl 
ing et al., 2007; Roach, Heron, \& McGraw, 2006; Sato, Toyoizumi, \& Aihara, 2007; Shams, Ma, \& Beierholm, 2005; Wozny, Beierholm, \& Shams, 2008), however, have concluded that sensory integration is partial rather than complete. That is, the sum of the weights for the modalities to be integrated is less than one, and the information of each modality remains individually accessible when all are combined. To accommodate partial integration, Bresciani et al. (2006) developed a Bayesian sensoryintegration scheme that allows incomplete integration. The scheme combines the observational variances of the individual sensory signals (the likelihood function) with prior knowledge about their coupling strength (the prior) to infer the most probable perceptual interpretation (the posterior) of the multisensory information. The freemodel parameter coupling prior distinguishes the model of Bresciani et al. (2006) from those of Andersen et al. (2005) and is functionally equivalent to the prior matrix in Shams et al. (2005). On top of this perceptual level, they assume a decision level that includes a cost-benefit function, which can be goal and task dependent (not implying a conscious process).

Until now, the models that have been mentioned have been validated mostly with experimental paradigms in which the participants' task was to ignore stimuli in the "irrelevant" channel (cued conditions). This was done to show that multimodal integration can occur even if observers want to prevent it. In cued conditions, however, the strength of the integration and the ability to ignore a channel are confounded. Others (Körding et al., 2007; Wozny et al., 2008) have studied conditions in which participants reported on all the modalities that were involved and hence were not cued to ignore any. Because these experiments were designed for other purposes, they did not compare these conditions with cued conditions.

We explicitly distinguish between two effects: (1) (bottom-up) channel-interference effects occurring in situations in which both channels are attended to and (2) (top-down) channel-suppression effects that result from selective attendance to the task-relevant channel and ignoring of the irrelevant.

To disentangle these two effects, we carried out an experiment in which participants were exposed to sequences of visual flashes and tactile taps in two conditions. In one condition, they were instructed - before the stimuli were presented - to report the number of events in a particular channel and to ignore those in the other (i.e., the traditional paradigm in which channel interference and suppression effects are combined). In a second condition, they were instructed on which channel to report only after the presentation; therefore, they could not ignore a channel (i.e., channel interference only). By comparing the results, we were able to quantify to what extent sensory interference and selective attention influence whole or partial perceptual integration.

We hypothesized that fission and fusion effects in bimodal counting tasks would be stronger when both sensory channels received equal or natural attention than when a single channel was ignored.
The numerosity-judgment paradigm allowed us to compare our results with those of Bresciani et al. (2006), Shams et al. (2005), and Andersen et al. (2005), who have all tested their sensory-integration models mainly for multimodal numerosity judgments.

\section{METHOD}

\section{Main Experiment}

Participants. Thirty-two right-handed, paid volunteers (16 female, 16 male; mean age $=21.5$ years) participated in the experiment. Participants were naive as to the purpose of the experiment, had normal or corrected-to-normal vision, and reported no history of sensory-motor disorder.

Setup. Participants were seated at a distance of $70 \mathrm{~cm}$ from a computer monitor in a dimly illuminated room. They fixated their view on a fixation cross on the monitor. A white LED $(\varnothing=5 \mathrm{~mm})$ was attached to the monitor at an eccentricity of $8^{\circ}$ under the fixation cross.

The right arm was comfortably placed on an armrest, and the index finger was positioned on a tactile device (B\&K mini-shaker 4810). Participants used the numeric keypad of a keyboard with their left hand to report the number of events that they perceived. In order to prevent participants from hearing the weak sound of the tactile device, they wore a sound-dampening headphone (Bilsom 747), and a set of speakers provided pink noise during the experiment.

Stimuli. The stimuli consisted of all nine combinations of series of one, two, or three flashes and one, two, or three taps. The first flash and tap were always delivered simultaneously $( \pm 1 \mathrm{msec})$. Multiple pulses had a 70-msec stimulus onset asynchrony. Flashes were produced by an LED $\left(80 \mathrm{~cd} / \mathrm{m}^{2}, 20 \mathrm{KHz}\right)$. Taps were generated by the mini-shaker in the form of a $100-\mathrm{Hz}$ sine wave, which indented the skin by $0.08 \mathrm{~mm}\left(12.5 \mathrm{~m} / \mathrm{sec}^{2}\right)$. Both taps and flashes had a duration of $10 \mathrm{msec}$ and were well above threshold.

Conditions. Two conditions were tested: cue (participants were told to report either flashes or taps before the stimulus was presented) and no cue (participants were told to report either flashes or taps after the stimulus was presented). Please note that the term "no cue" refers to the absence of a cue before stimulus presentation.

All stimulus combinations were presented 20 times in random order for each task (report flashes or report taps) and each condition (cue or no cue), yielding 720 stimulus presentations (trials) per participant. The 360 trials for the cue condition were pseudorandomly distributed across three blocks of 120 trials each. The no-cue trials were distributed similarly across three blocks. The order of the six blocks was balanced across participants.

Procedure. Before the start of the experiment, participants received a maximum of five tactile and five visual practice blocks consisting of 30 unimodal stimuli each (either flashes or taps). After each practice block, participants were informed about the percentage of their judgments that were correct. As soon as a participant had scored more than $80 \%$ correct in a tactile and visual block, he or she started the experiment. One participant did not meet the criteria after five tactile blocks and was excluded.

At the start of the experiment, participants received instructions, and before each block, they were told whether it was a cued block or not. One block lasted approximately $15 \mathrm{~min}$, including a short break after 60 trials. Participants were allowed to take a more extensive break between blocks.

In the cue condition, the fixation cross was replaced with the word "flash" or "tap," indicating the modality to be reported, followed by the stimulus presentation and a question mark, indicating that the number of events in the cued modality had to be typed in by the participant. In the no-cue condition, the monitor displayed only an exclamation mark, followed by the stimulus presentation. Directly after the presentation of the stimulus, the fixation cross was replaced 
by either the word "flash" or "tap," indicating that either the number of flashes or the number of taps had to be reported. When participants failed to give a response within $5 \mathrm{sec}$, the next trial was presented.

An experiment leader observed the participant during the session to ensure that the participant followed the instructions.

Definitions. Participants always reported the number of events in only one of two sensory channels (the relevant channel) making the other channel irrelevant for the task.

Each trial was coded as fusion (the number of events in the irrelevant channel was lower than the number of events in the relevant channel), fission (the number of events in the irrelevant channel exceeded the number of events in the relevant channel), or congruent (the number of events in the irrelevant channel was equal to the number of events in the relevant channel).

\section{Control Experiment}

A useful discussion of our findings in the context of current sensory-integration models requires a comparison of the judgments of unisensory and congruent stimuli.

In a separate control experiment, participants judged the numerosity of unisensory stimuli that contained either flashes (one, two, or three) or taps (one, two, or three). The 12 participants were a subset of the participants in the main experiment.

All six stimuli were presented 20 times in random order for each condition, similar to the presentation of congruent stimuli in the main experiment; thus, each participant judged two blocks of 120 stimulus presentations each. The setup, procedure, and stimuli were similar to those in the main experiment.

\section{RESULTS}

\section{Main Experiment}

Trials for which participants had failed to give a response $(70$, or $0.3 \%)$ were removed. Trials for which participants did not respond with " 1 ," "2," or " 3 " (62, or $0.3 \%$ ) were also removed, since we considered these to be typing errors. For the remaining 22,188 responses, we computed the bias as the participants' response minus the correct response.

The average bias of all responses was $-0.17(S D=$ 0.53 ), indicating that participants had a general tendency to underestimate the number of events that were presented. This bias differed significantly from zero [onetailed $t$ test: $t(30)=-1.76, p<.05]$.

For congruent stimuli, the average bias was -0.20 $(S D=0.49)$ for reporting flashes and $-0.10(S D=0.42)$ for reporting taps. These biases differed significantly from each other [matched-pair $t$ test: $t(30)=-4.12, p<.001$ ] The specific response values that were reported for congruent stimuli are given in Table 1.

The data were further analyzed with a repeated measures ANOVA with the following design: condition (cue

Table 1

Responses (Means and Standard Deviations) for the Three Congruent Stimulus Combinations (One Flash and One Tap, Two Flashes and Two Taps, Three Flashes and Three Taps) for the Report Flashes Task and Report Taps Task

\begin{tabular}{|c|c|c|c|c|}
\hline \multirow{3}{*}{$\begin{array}{c}\text { Congruent } \\
\text { Number of } \\
\text { Events }\end{array}$} & \multicolumn{4}{|c|}{ Task } \\
\hline & \multicolumn{2}{|c|}{ Report Flashes } & \multicolumn{2}{|c|}{ Report Taps } \\
\hline & $M$ & $S D$ & $M$ & $S D$ \\
\hline One & 1.02 & 0.17 & 1.02 & 0.15 \\
\hline Two & 1.82 & 0.50 & 2.05 & 0.39 \\
\hline Three & 2.54 & 0.57 & 2.64 & 0.51 \\
\hline
\end{tabular}

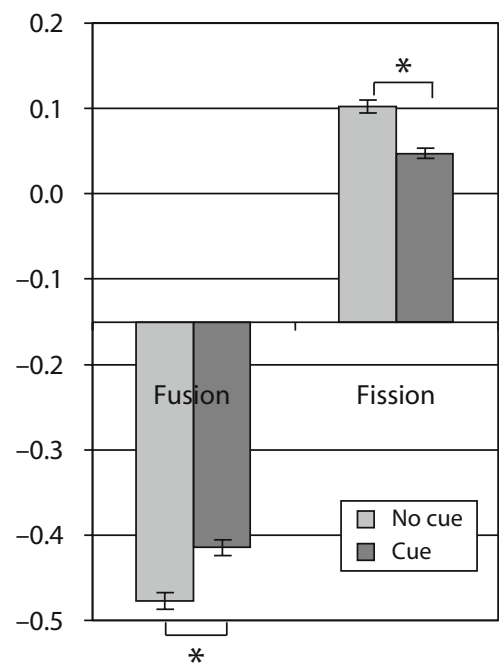

Figure 1. The average response biases for fusion trials (left) and fission trials (right). The light and dark bars show responses for the no-cue and cue conditions, respectively, relative to the average response for congruent trials $(-0.15) . \quad{ }^{*} p<.001$.

or no cue $) \times$ task (report flashes or report taps) $\times$ type $($ fusion or fission). If appropriate, we used a post hoc Tukey HSD test.

We found a significant main effect of task $[F(1,30)=$ $5.20, p<.05]$. The average response across fission and fusion trials and conditions was significantly lower $(-0.28)$ for the report flash task than for the report taps task $(-0.15)$. Furthermore, we found a significant main effect of type $[F(1,30)=130.30, p<.001]$. The average response across tasks and conditions was significantly lower for fusion trials $(-0.45)$ than for fission trials $(0.08)$.

The main effect of condition $[F(1,30)=0.06, p=.81]$ was not significant (note that biases for fission and fusion trials can cancel each other out, because they have opposite signs); however, the condition $\times$ type interaction effect was significant $[F(1,30)=11.87, p<.001]$ and is depicted in Figure 1.

When averaged across tasks, fission effects were stronger for the no-cue condition than for the cue condition ( 0.10 vs. $0.05 ; p<.001)$. Even so, fusion effects were stronger for the no-cue condition than for the cue condition $(-0.48$ vs. $-0.42 ; p<.001)$.

Another interesting significant effect was the condition $\times$ task $\times$ type interaction $[F(1,30)=4.25, p<.05]$. This interaction is depicted in Figure 2, in which the bars are plotted relative to the overall response bias for congruent trials in each task $(-0.20$ for report flashes and -0.10 for report taps). The post hoc test showed that the increase of fusion and fission effects for no-cue versus cue conditions was significant for the report taps task $(p<.001)$ but not for the report flashes task ( $p=.45$ and $p=.10$ ).

\section{Control Experiment}

Table 2 shows the unisensory response values of the control experiment.

A pairwise comparison between the congruent stimuli of the main experiment and the unisensory condition for 
Report Flashes

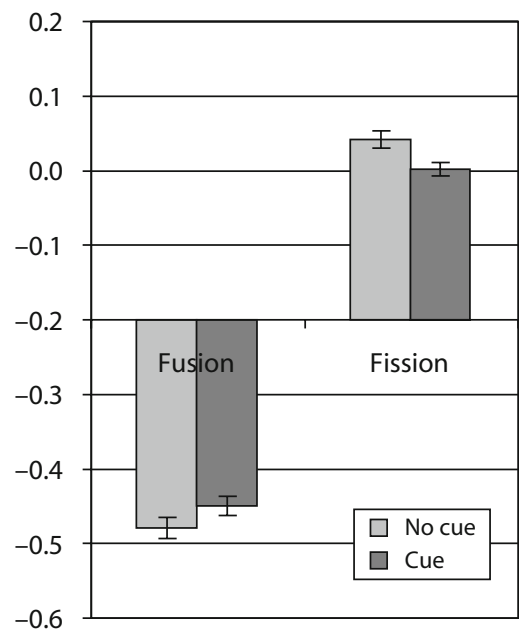

Report Taps

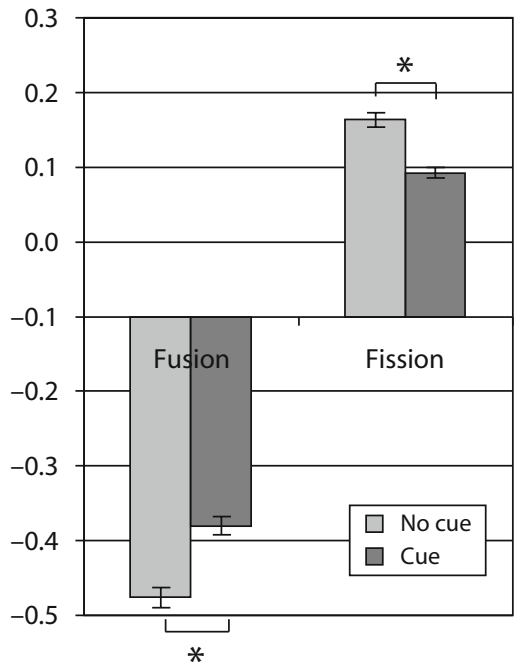

Figure 2. The average response biases for the report flashes task (left panel) and report taps task (right panel). Each panel shows the biases for fusion trials (bars on the left) and fission trials (bars on the right). The light bars are for the no-cue condition, and the dark bars are for the cue condition. The bars for each task are plotted relative to the average response for congruent trials for that task. $\quad " p<.001$.

each number of events yielded no significant differences for the judgment of the number of flashes $(p=.85, .65$, and .88 for one, two, and three flashes, respectively). Similarly, we found no significant differences between congruent and unisensory judgments of the number of taps $(p=.83, .30$, and .54 for one, two, and three taps, respectively).

\section{DISCUSSION}

In general, our findings of touch-induced visual illusions and vision-induced touch illusions in the cue condition are well in line with results that were reported by Violentyev et al. (2005) and Bresciani et al. (2006). We found substantial fission and fusion effects. The significant main effect of type shows that response biases were on average 0.52 lower for fusion than for fission trials. This is a substantial effect, given that if the irrelevant channel had fully "captured" the relevant channel, the average response bias would differ by 2.67 for fusion and fission trials $(-1.33$ for fusion and +1.33 for fission).

Interestingly, we also found a response bias when the number of flashes equaled the number of taps, indicating a general tendency to underestimate the number of

Table 2

Responses (Means and Standard Deviations) for the Three Unisensory Stimuli (One, Two, or Three Flashes; One, Two, or Three Taps)

\begin{tabular}{cccccc}
\hline \multirow{2}{*}{$\begin{array}{c}\text { Number of } \\
\text { Events }\end{array}$} & \multicolumn{2}{c}{ Flashes } & & \multicolumn{2}{c}{ Taps } \\
\cline { 2 - 3 } \cline { 5 - 6 } & $M$ & $S D$ & & $M$ & $S D$ \\
\hline One & 1.01 & 0.11 & & 1.01 & 0.10 \\
Two & 1.74 & 0.54 & & 1.91 & 0.40 \\
Three & 2.51 & 0.54 & & 2.53 & 0.55 \\
\hline
\end{tabular}

events. For such congruent stimuli, we found an average response bias of -0.2 for reporting flashes and -0.1 for reporting taps. With respect to systematic errors, therefore, counting taps is more accurate than counting flashes. This is in agreement with the findings that were reported by Philippi, van Erp, and Werkhoven (2008).

The control experiment showed that multimodal congruent-numerosity judgments did not differ significantly from the unimodal judgments. This is consistent with data from most previous studies, all of which reported insignificant differences (Andersen et al., 2004; Violentyev et al., 2005; Wozny et al., 2008). It should be noted that Philippi et al. (2008) - who studied uni-, bi-, and trimodal temporal-numerosity judgments for a wide range of ISIs (20-320 msec) and number of events (2-10) - did find significant differences in bias for uniand multimodal conditions; however, they did not report significant differences for the vision and touch combination at the ISIs that were tested here.

Unlike previous studies (Bresciani et al., 2006), we did not find a decrease in $S D$ (or variance) for multimodal (congruent) stimuli. In fact, multimodal presentation slightly increased the $S D$ of responses ( $4 \%$ for taps, $0 \%$ for flashes). This is consistent with Philippi et al. (2008), who found that the variance of congruent stimuli decreased relative to unisensory stimuli for interstimulus intervals (ISIs) of $160 \mathrm{msec}$ and larger, but that it increased for shorter ISIs.

The effect of a manipulation of attention on the variance of judgments can be understood by assuming that the amount of attention that is allocated to a sensory modality determines the variance of that modality and consequently its weight in (late) integration models (Andersen et al., 2004; Helbig \& Ernst, 2008). If resources that are 
related to attention are amodal and limited, a focus of attention will increase the variance of the relevant channel and decrease the variance of the irrelevant channel. The relative change of the variances of the modalities that are involved affects the integration process and thus the judgment bias.

A separate issue is how the multimodal sensoryintegration process affects the variance of numerosity judgments. MLE-like integration schemes predict lower variances for congruent judgments than for unisensory judgments; however, the variance of the congruent judgments can increase if the variances that are input to the integration have been increased by divided attention that results from multimodal presentation. The combined effects may explain the variances that we observed for multimodal stimuli.

\section{Disentangling Bottom-Up Interference and Top-Down Attentive Suppression}

Unlike in previously reported studies, our method allowed us to disentangle the integration effect into bottomup interference effects (the mutual influence of sensory modalities when both are attended) and the effect of selective attention to a single modality that is assumed to (partly) suppress the sensory interference process. Previously reported asymmetric response biases may in fact have originated from these combined effects and not purely from asymmetries in (bottom-up) sensory-interference effects. For example, the conclusion that vision influences touch more than touch influences vision in cue conditions (Guest \& Spence, 2003) may have been caused by the fact that it is easier to attentively suppress touch than to do so for vision, even when the underlying sensory-integration effects are symmetric. In fact, asymmetries in attentive processes have been reported by Spence, Shore, and Klein (2001) and by Spence, Nicholls, and Driver (2001), showing that attention can be directed to a particular sensory modality and that it is more difficult to shift attention away from the tactile modality than from vision or audition.

When discussing the size of sensory-integration effects, we must take into account the effect of fission as well as fusion. To have a single measure for the influence of one modality on another, we express the sensoryintegration effect as the sum of the absolute fission and fusion effects. This integration effect is assumed to be maximal when no sensory channel is suppressed - that is, in the no-cue condition. This maximal (bottom-up) integration effect was 0.52 for the report flashes task and 0.64 for the report taps task; thus, the bottom-up interference effect of vision on touch was stronger than the influence of touch on vision.

We have hypothesized that the bottom-up interference effect is reduced by a top-down attentive suppression of the irrelevant channel. Our results confirm this hypothesis. In the cue condition, the integration effect for the report flashes task reduced substantially, from 0.52 to 0.45 $[t(30)=1.95, p<.06]$; that is, the favorable effect of suppressing irrelevant tactile information equaled $13 \%$. For the report taps task, the integration effect reduced sig- nificantly, from 0.64 to $0.47[t(30)=3.59, p<.01]$; that is, the favorable effect of suppressing irrelevant visual information equaled $27 \%$. This suggests that it is easier to suppress vision than it is to suppress touch.

Spence, Nicholls, and Driver (2001) and Guest and Spence (2003) also found an asymmetry between cued vision-induced touch and touch-induced vision tasks and suggested that people find it hard to shift attention away from the tactile modality. An imbalance between the strong influence of touch on vision and the weak influence of vision on touch was also found by Bresciani et al. (2006). Furthermore, Bresciani, Dammeier, and Ernst (2008) found that vision was the modality most susceptible to being biased in trimodal event-counting tasks and the least efficient in biasing the other two modalities. Note, however, that in these studies, bottom-up integration effects were confounded with attentive suppression, in contrast to our study. We found that the bottom-up sensory interference effect of vision was stronger, but that vision was also more easily suppressed by top-down selective attention.

In our study, the visual and tactile stimuli were not colocated; therefore, a focusing of attention on a particular sensory modality was in principle confounded with a focusing of attention on a particular location. Focusing of attention to a particular location, however, does not seem to explain the differences in effect size that we observed between touch-induced vision and vision-induced touch in our cue condition. One can argue that it is easier to suppress a visual stimulus than to suppress a tactile stimulus, but it seems arbitrary to assume that suppressing one location is easier than suppressing another location. Also, our results for the cue condition without spatial alignment are well in line with those from experiments in which visual and tactile stimuli were co-located (e.g., Bresciani et al., 2006). We are confident, therefore, that the attentive process that was studied here is a focus on modality, not on location.

\section{Temporal Versus Spatial Numerosity Estimation}

Gallace, Tan, and Spence (2007) were the first to report on multisensory spatial numerosity judgments. In spatial numerosity judgment, stimuli are presented simultaneously at multiple locations, as opposed to sequentially at a single location, which occurs in temporal numerosity judgments. Participants were instructed to count the total number of pulses that were presented, summed over the tactile and visual modalities (i.e., the two modalities did not provide redundant information). They found that the amount of underestimation was larger for multisensory presentation than for unisensory presentation, which was hypothesized to result from amodal multisensory limitations in spatial processing. Alternatively, the decrease in performance for bimodal presentations may be explained by assuming that participants could not suppress the integration of visual and tactile events.

\section{Sensory Memory Effects}

Theoretically, the effects of attention that have been discussed in our study could be the result of a stimulus- 
processing mechanism that operates on a high-capacity sensory-storage system (Bliss, Crane, Mansfield, \& Townsend, 1966; Gallace, Tan, Haggard, \& Spence, 2008; Griffin \& Nobre, 2003; Matsukura, Luck, \& Vecera, 2007; Miles \& Borthwick, 1996; Sperling, 1960) in which stimuli are retained for a brief interval of time. That is, independently of whether the task cue is presented just before or just after the stimulus presentation, the judgment can be based on representations of the stimulus in unimodal or multimodal sensory memories allowing various sorts of (late) top-down attentional or cognitive manipulations.

The fact that we did find differences for the cue and no-cue conditions, however, indicates that the attentional process is affected in substantial part by cue manipulations and is not solely a higher order independent process in sensory memory. Even if higher order sensory memory effects remain (which we cannot exclude), furthermore, we have succeeded in revealing cue-dependent attentional processes and in showing the need to unconfound bottomup integration and top-down suppression effects when trying to understand sensory-integration processes. We would expect to find even stronger bottom-up sensoryintegration effects if we manipulated sensory memory to rule out remaining top-down sensory-memory effects.

A strongly related issue is the possible existence of a visual interference effect between sensory memory and the visual cue that was presented right after the stimulus in the no-cue conditions. Such an interference effect is most likely to increase the variance of the visual signal. In any weighted integration scheme, this would make the visual signal less influential; thus, we would expect stronger fission and fusion effects for the report flashes task in the no-cue condition than in the cue condition, which is indeed what we observed (though it was not significant) in Figure 2 (left panel). Similarly, we would also expect that for the report taps task the visual signal would be less influential in the no-cue condition than in the cue condition, yielding stronger fission and fusion effects in the cue condition. Figure 2 (right panel), however, shows the (significant) opposite of this prediction. Consequently, we conclude that the hypothetical visual interference effect in the no-cue condition is either insignificant or nonexistent.

\section{Model Implications}

Although the primary purpose of our study was to disentangle bottom-up sensory integration and top-down attentional effects, we will now discuss some model implications of our results.

MLE schemes (Andersen et al., 2005) are based on a complete weighted integration of sensory signals in which the weights are inversely proportional to the variances of the unimodal signals. Consequently, the differences in biases (and thus weights) for the different tasks in our study (report flashes and report taps) can be explained only if attentive suppression of the irrelevant channel can change the weights (and thus the variances) of the unimodal stimuli. This would mean that sensory integration is not an entirely preattentive process. It should be noted that the nature of the sensory-integration process may depend strongly on the amount of spatial and temporal conflict between the unimodal signals (for an extensive review of this issue, see Helbig \& Ernst, 2008).

Although MLE may explain our results for the cue condition by accepting that sensory integration is not entirely preattentive, it cannot explain our findings for the no-cue condition. In the no-cue condition, participants did not know the task and could not selectively pay attention to one specific channel. Consequently, MLE cannot predict the different biases for the report flashes and report taps tasks, as we found in our experiments.

To understand the different response biases that were found for the different tasks and conditions, we adopted the Bayesian sensory-integration model that was developed by Bresciani et al. (2006). In this model, the most probable perceptual interpretation (posterior) of the multisensory information is determined on the basis of the individual sensory signals (the likelihood functions) and prior knowledge about their coupling strength (the coupling prior). The fact that sensory signals do not fuse completely and remain individually accessible allows for different responses in our cue and no-cue conditions. The mutual influence between the sensory channels is reflected in the coupling strength, which is a function of the coupling prior and (changing) likelihoods and thus is sensitive to attentional effects.

The coupling strength can be calculated for both conditions on the basis of our fission and fusion responses. Following Bresciani et al. (2006), the coupling strength $L$ is defined as the sum of the relative change $\left(\Delta_{V}\right)$ in the number of flashes that were perceived for the report flashes task and the relative change $\left(\Delta_{T}\right)$ in the number of taps that were perceived for the report taps task:

$$
L=\Delta_{V}+\Delta_{T}=\left|\frac{v-\hat{v}}{\hat{t}-\hat{v}}\right|+\left|\frac{t-\hat{t}}{\hat{v}-\hat{t}}\right|,
$$

where $\hat{v}$ and $\hat{t}$ are the number of flashes and taps reported for unimodal stimuli $V$ and $T$, respectively; $v$ is the number of flashes reported for a bimodal stimulus and report flashes task; and $t$ is the number of flashes perceived for a bimodal stimulus and report taps task. Because the unimodal and congruent response biases do not differ significantly (see the control experiment), we can also take the congruent response biases for estimating $\hat{v}$ and $\hat{t}$. A linear regression of the averaged report flashes and report taps responses (see Table 1) yields the following: $\hat{v}(V)=$ $0.28+0.76 V$ and $\hat{t}(T)=0.28+0.81 T$.

The coupling strength $L$ can be computed from the fission and fusion data that are given in Figure 2 for both conditions. For example, in the report flashes task, the fission bias $v-\hat{v}$ was 0.24 for the no-cue condition. The fission stimuli consisted of the combinations one flash and two taps, one flash and three taps, and two flashes and three taps, yielding an average stimulus conflict of 1.33 , an average $\hat{t}-\hat{v}=1.15$, and $\Delta_{V}=.21$. The same stimuli were tested as fusion stimuli for the report taps task and no-cue condition, yielding $\Delta_{T}=.33$. We found 
the coupling strength by adding $\Delta_{V}$ and $\Delta_{T}: L=.54$ for the no-cue condition when $V<T$. When $V>T$, we found $L=.57$ for the no-cue condition (the sum of $\Delta_{V}=.29$ and $\Delta_{T}=.28$ ). Analog calculations for the cue condition yielded $L=.42$ when $V<T$, and $L=.46$ when $V>T$. Selective attention thus reduced the coupling strength from .54 to $.42(22 \%)$ when $V<T$, and from .57 to .46 (19\%) when $V>T$.

A computation of $L$ values per participant yielded the standard deviation of the $L$ distributions. For no-cue conditions, these were 0.29 for $L$ when $V<T$, and 0.42 for $L$ when $V>T$. For cue conditions, these were 0.25 for $L$ when $V<T$, and 0.46 for $L$ when $V>T$. A repeated measures ANOVA showed that the effect of condition on $L$ was significant $[F(1,30)=11.97, p<.01]$.

At this point, we can conclude that MLE fails and that our results can only be understood by a Bayesian integration model allowing incomplete integration, reflected in a coupling strength that is smaller than one. By comparing the coupling strengths for the cue and no-cue conditions, we find that coupling strength is reduced by selectively suppressing the irrelevant channel, indicating that the coupling strength is sensitive to attentional effects.

Finally, we want to understand why we found a systematic underestimation of the number of events, even for congruent stimuli ( -0.20 for flashes, and -0.10 for taps). A possible general explanation for the underestimation of the number of events is that the variance increased with the number of events (see Table 1). A constant ratio between the response value and its standard deviation (signal-tonoise ratio) would indicate a Weber law, and the inverse ratio is called the Weber fraction (Fechner, 1860). For the congruent data in our study, the Weber fractions varied between $15 \%$ and $27 \%$.

Now, assume, for simplicity, a sensory-integration scheme such as MLE in which the integration weight of a sensory signal $S$ is inversely proportional to its standard deviation. Using this scheme, we can consider an example of a bimodal congruent stimulus consisting of $S$ flashes and $S$ taps. If the internal noisy "measurements" of the signals are $(1-\delta) S$ and $(1+\delta) S$ for flashes and taps, respectively, and we weight them with weight factors that are proportional to their variances $[(1-\delta) S]^{-2}$ and $[(1+\delta) S]^{-2}$, respectively (similar Weber laws for both signals), the estimation of $S^{\prime}$ on the basis of the two signals would be:

$$
S^{\prime}=\frac{1-\delta^{2}}{1+\delta^{2}} S
$$

More specifically, for a two-flash, two-tap stimulus $(S=2)$ and $\delta=0.25$ (the internal measures are 1.5 flashes and 2.5 taps), the weighted estimation would be $S^{\prime}=1.76$.

More generally, the number of events will be underestimated if the variance of a signal increases with the signal, consistent with our findings. Interestingly, the notion of reliability of a sensory channel in MLE schemes is determined exclusively by its signal-to-noise ratio or variance (for a review, see Ernst \& Bülthoff, 2004). Consequently, a sensory channel with a high signal-to-noise ratio is called a reliable channel, even when it shows a strong systematic error. It is in principle impossible, however, to take response biases into account in ideal observer models. Any observation system having knowledge about its systematic error would be able to correct its response accordingly and eliminate the systematic error by doing so.

\section{Conclusion}

We disentangled bottom-up sensory interference effects and top-down attentive effects for multisensory visualtactile integration. Our results show that the bottom-up sensory interference effect of vision is stronger, but that vision is also more easily suppressed by top-down selective attention. The results are compatible with a partial (Bayesian) sensory-integration model with an average coupling strength of .56, which is reduced approximately $20 \%$ by selective attention.

\section{AUTHOR NOTE}

Correspondence concerning this article should be addressed to P. J. Werkhoven, Department of Information and Computing Sciences, Utrecht University, Padualaan 14, de Uithof, 3584 CH Utrecht, The Netherlands (e-mail: peter.werkhoven@tno.nl).

\section{REFERENCES}

Andersen, T. S., Tirppana, K., \& Sams, M. (2004). Factors influencing audiovisual fission and fusion illusions. Cognitive Brain Research, 21, 301-308

Andersen, T. S., Tirppana, K., \& Sams, M. (2005). Maximum likelihood integration of rapid flashes and beeps. Neuroscience Letters, 380, 155-160.

Bliss, J. C., Crane, H. D., Mansfield, P. K., \& Townsend, J. T. (1966) Information available in brief tactile presentations. Perception \& Psychophysics, 1, 273-283.

Bresciani, J.-P., Dammeier, F., \& Ernst, M. O. (2006). Vision and touch are automatically integrated for the perception of sequences of events. Journal of Vision, 6, 554-564.

Bresciani, J.-P., DAmmeier, F., \& ERnst, M. O. (2008). Trimodal integration of visual, tactile and auditory signals for the perception of sequences of events. Brain Research Bulletin, 75, 753-760.

Bresciani, J.-P., Ernst, M. O., Drewing, K., Bouyer, G., Maury, V., \& Kheddar, A. (2005). Feeling what you hear: Auditory signals can modulate tactile tap perception. Experimental Brain Research, 162, 172-180.

Courtney, J. R., Motes, M. A., \& Hubbard, T. L. (2007). Multi- and unisensory visual flash illusions. Perception, 36, 516-524.

Ernst, M. O., Bresciani, J.-P., Drewing, K., \& Bülthoff, H. H. (2004). Integration of sensory information within touch and across modalities. In Proceedings of the 2004 International Conference on Intelligent Robots and Systems (IROS 2004), 1-2 (10 2004).

ERnst, M. O., \& Bülthoff, H. H. (2004). Merging the senses into a robust percept. Trends in Cognitive Sciences, 8, 162-169.

Fechner, G. T. (1860). Elemente der Psychophysik. Leipzig: Breitkopf \& Härtel.

Gallace, A., Tan, H. Z., Haggard, P., \& Spence, C. (2008). Shortterm memory for tactile stimuli. Brain Research, 1190, 132-142.

Gallace, A., TAN, H. Z., \& Spence, C. (2007). Multisensory numerosity judgments for visual and tactile stimuli. Perception \& Psychophysics, 69, 487-501.

Griffin, I. C., \& Nobre, A. C. (2003). Orienting attention to locations in internal representations. Journal of Cognitive Neuroscience, 15, 1176-1194.

Guest, S., \& Spence, C. (2003). Tactile dominance in speeded discrimination of textures. Experimental Brain Research, 150, 201-207.

Helbig, H. B., \& ERnst, M. O. (2008). Visual-haptic cue weighting is independent of modality-specific attention. Journal of Vision, 8, 1-16.

Hötting, K., \& RöDER, B. (2004). Hearing cheats touch, but less in congenitally blind than in sighted individuals. Psychological Science, 15, 60-64. 
Körding, K. P., Beierholm, U. [R.], Ma, W. J., Quartz, S., TenenBaUm, J. B., \& Shams, L. (2007). Causal inference in multisensory perception. PLOS ONE, 2, e943.

Matsukura, M., Luck, S. J., \& Vecera, S. P. (2007). Attention effects during visual short-term memory maintenance: Protection or prioritization? Perception \& Psychophysics, 69, 1422-1434.

Miles, C., \& BoRTHWicK, H. (1996). Tactile short-term memory revisited. Memory, 4, 655-668.

PhilipPI, T., van ERP, J. B. F., \& Werkhoven, P. J. (2008). Multisensory temporal numerosity judgment. Brain Research, 1242, 116-125. doi:10.1016/j.brainres.2008.05.056

Roach, N. W., Heron, J., \& McGraw, P. V. (2006). Resolving multisensory conflict: A strategy for balancing the costs and benefits of audio-visual integration. Proceedings of the Royal Society B, 273, 2159-2168.

Sato, Y., Toyoizumi, T., \& Ainara, K. (2007). Bayesian inference explains perception of unity and ventriloquism aftereffect: Identification of common sources of audiovisual stimuli. Neural Computation, 19, 3335-3355.

Shams, L., Kamitani, Y., \& Shimojo, S. (2000). What you see is what you hear. Nature, $\mathbf{4 0 8}, 788$.
Shams, L., Kamitani, Y., \& Shimojo, S. (2002). A visual illusion induced by sound. Cognitive Brain Research, 14, 147-152.

Shams, L., MA, W. J., \& Beierholm, U. [R.] (2005). Sound-induced flash illusion as an optimal percept. NeuroReport, 16, 1923-1927.

Spence, C., Nicholls, M. E. R., \& Driver, J. (2001). The cost of expecting events in the wrong sensory modality. Perception \& Psychophysics, 63, 330-336.

Spence, C., Shore, D. I., \& Klein, R. M. (2001). Multisensory prior entry. Journal of Experimental Psychology: General, 130, 799-832.

SPERLING, G. (1960). The information available in brief visual presentations. Psychological Monographs: General \& Applied, 74, 1-29.

Violentyev, A., Shimojo, S., \& Shams, L. (2005). Touch-induced visual illusion. NeuroReport, 16, 1107-1110.

WELCH, R. B., \& WARREN, D. H. (1980). Immediate perceptual response to intersensory discrepancy. Psychological Bulletin, 88, 638-667.

WoZny, D. R., BeIERHOLM, U. R., \& ShAMs, L. (2008). Human trimodal perception follows optimal statistical inference. Journal of Vision, $\mathbf{8}$, $1-11$.

(Manuscript received December 14, 2008; revision accepted for publication July 5, 2009.) 